\title{
Interferometric Imaging of Geo-synchronous Satellites with Ground-based Telescopes
}

\author{
John Young \\ Cavendish Laboratory \\ Cambridge CB3 OHE, UK \\ +441223 337347 \\ jsy1001@cam.ac.uk
}

\author{
Chris Haniff \\ Cavendish Laboratory \\ Cambridge CB3 OHE, UK \\ +441223 337307 \\ cah@mrao.cam.ac.uk
}

\author{
David Buscher \\ Cavendish Laboratory \\ Cambridge CB3 OHE, UK \\ +441223 337302 \\ dfb@mrao.cam.ac.uk
}

\begin{abstract}
Inteferometric imaging with arrays of telescopes is now a routine technique in optical/infrared astronomy, delivering images with nano-radian resolution. Sensitive next generation arrays, such as the Magdalena Ridge Observatory Interferometer, thus offer a potentially important capability for space situational awareness, allowing satellites in geo-synchronous Earth orbit (GEO) to be inspected after launch. A critical factor for such observations is a lack of short spacings between the telescopes comprising the interferometric array. Large GEO satellites are frequently $20-30 \mathrm{~m}$ in size, hence ideally the shortest spacings used for the observations should be between $1.2-1.8 \mathrm{~m}$ for observations at $1 \mu \mathrm{m}$ wavelength. These cannot easily be realised with existing separated-element arrays. In this paper we explore the approach of combining observations from a separated-element interferometer with interferometric data obtained by optical masking of a "single-dish" telescope. We present simulations that demonstrate that substantial improvements in image fidelity can be achieved in this way. Two shapes of generic satellite shape are investigated, intended to be representative of the majority of bright GEO targets, and the efficacy of the method is assessed for these different target shapes and differing brightness levels.
\end{abstract}

\section{TABle of Contents}

1 Introduction $. . . \ldots \ldots \ldots \ldots \ldots \ldots \ldots \ldots \ldots, 1$

2 IMAGING SATELLITES WITH LONG-BASELINE ARRAYS $\ldots \ldots \ldots \ldots \ldots \ldots \ldots \ldots \ldots \ldots \ldots \ldots \ldots, 2$

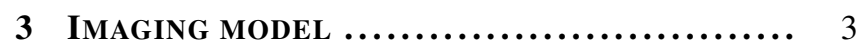

4 Simulations............................... 4

5 RESUltS .................................... 5

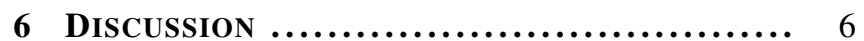

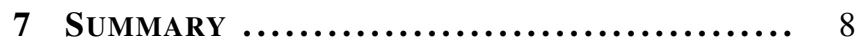

ACKNOWLEDGMENTS ........................ 8

REFERENCES ............................... 8

BIOGRAPHIES ............................... 8

\section{INTRODUCTION}

The increasingly large volume of commercial and military hardware in geo-synchronous Earth orbit (GEO) has led to a premium on methods for reliable optical/infrared (IR) imaging of these multi-billion dollar value assets. Not only would high angular resolution imaging be of use for diagnostic evaluation, e.g. for confirming the successful deployment of solar panels or high-gain antennae, but such a capability might also allow for the monitoring of the local environments of such satellites and the detection of potentially harmful local space debris.

978-1-4673-1813-6/13/\$31.00 (C)2013 IEEE.

${ }^{1}$ IEEEAC Paper \#2257, Version 3.0, Updated 04/01/2013.
However, the current capabilities of the largest ground-based telescopes fall well below what is necessary for a useful geo-synchronous imaging capability. For example, a $10 \mathrm{~m}$ telescope operating with a perfect adaptive optics (AO) system would have a diffraction limited resolution in the nearinfrared $H$ band (centred at $1.65 \mu \mathrm{m}$ ) of roughly $7 \mathrm{~m}$. This is much larger than a typical geo-synchronous bus diameter but is able to resolve the longest dimension of large solar panels. The next-generation of 30-40 m-class ground-based astronomical telescopes would conceivably be able to deliver a more valuable capability, i.e. with resolutions at the few metre level, but the projected timelines for these projects suggest that such diffraction-limited imaging will not occur until the post-2020 timeframe.

An alternative strategy is to utilise aperture synthesis methods. Long baseline optical/IR interferometric arrays, such as the CHARA, VLTI and NPOI telescopes, can now routinely deliver images of astronomical targets with 5 nanoradian angular resolution. In geo-synchronous orbit this corresponds to a spatial scale of only $18 \mathrm{~cm}$. A key shortcoming of such arrays, though, has been their relatively poor sensitivity as compared to single dish telescopes. However, secondgeneration arrays such as the Magdalena Ridge Observatory Interferometer (MROI) [1], are expected to realise a limiting sensitivity some $10-100 \times$ better than existing arrays, allowing unprecedented access to a considerable fraction of the population of geo-synchronous targets.

In a recent paper [2] we presented a preliminary analysis of the capabilities of the MROI for geo-synchronous satellite imaging. While our sensitivity calculations suggested that as many as $50 \%$ of GEO targets would be detectable, the large linear sizes of most of these targets presented a challenge for interferometric imaging. Existing long-baseline arrays have such a high angular resolution that they "resolve out" the large scale structure in the targets, rendering reliable imaging difficult. While the use of a-priori information, such as image positivity, can help to mitigate some of the resulting imaging artefacts, a better approach may be to explicitly capture low angular resolution information on the target and then use this additional data in the interferometric image reconstruction process.

In this paper we explore such an approach, where data from a long baseline interferometer are combined with data secured contemporaneously with a separate large aperture (i.e. $10-\mathrm{m}$ class) AO-corrected telescope. A novel feature of our scheme is that we assume that the single telescope is used in an "nonredundant aperture-masked" mode, emulating the use of an interferometric array, though with baselines only extending to a maximum of set by the aperture diameter. This is a somewhat different approach to that of Mozurkewich et al. [3] who have investigated the use of a common-mount interferometer, i.e. a multi-element interferometric array mounted on a large 
$50 \mathrm{~m}$ diameter steerable platform, but with baselines ranging from a few metres to the maximum size of the platform.

We discuss the feasibility of such a hybrid "single telescope+long baseline array" scheme and present imaging simulations using a Maximum Entropy based algorithm that combines the sparse Fourier data measured by the longbaseline and single-dish interferometer arrays to recover high angular resolution images. Interestingly, we find that even a relatively small amount of shorter baseline data can improve the quality and reliability of the interferometric image reconstructions substantively.

In section 2 we present a brief review of the difficulties of GEO imaging, while in sections 3 and 4 we outline the implementation of the imaging and image recovery models we have used. Our results are presented in section 5 and are discussed in section 6. Finally, we summarise our key findings in section 7 .

\section{IMAGING SATELLITES WITH LONG-BASELINE ARRAYS}

The empirical rules of thumb established through years of radio interferometric imaging can be straightforwardly translated to the problem of interferometric imaging of geosynchronous satellites. As for all interferometric arrays, the interference signals secured on those telescopes with the longest telescope-to-telescope separations (a.k.a "baselines") will be sensitive to the smallest angular scales (or equivalently the highest spatial frequencies) in the target brightness distribution. Conversely, it will be the shortest interferometer baselines that probe the largest angular scales in the target.

In the $1.65 \mu \mathrm{m} H$ band window, a $1 \mathrm{~m}$ structure at geosynchronous altitude would require interferometer baselines at least as long as $59 \mathrm{~m}$ to resolve it fully. On the other hand, if the same target happened to have solar panels spanning $27 \mathrm{~m}$ - a median value for the GEO assets seen nowadays - data secured on baselines as short as $2 \mathrm{~m}$ would typically be required to reproduce these in any recovered image. Interferometric imaging studies typically aim to sample the 2-dimensional Fourier (or $u v$ ) plane with baselines ranging from some maximum, $B_{\max }$, to some minimum, $B_{\min }$, in as uniform a way as is reasonably possible. Under these circumstances, then one is usually able to recover an image with at least $N \times N$ independent resolution elements, where $N \simeq B_{\max } / B_{\min }$, as long as roughly this number of independent measurements of the $u v$ plane are secured. For the range of spatial scales outlined above, this would correspond to approximately 500 separate Fourier plane measurements.

The ability of existing synthesis arrays to secure such large datasets is an issue. An $N$-element array measures $N(N-$ 1) $/ 2$ independent Fourier data per spectral channel at any instant in time and so, for example, 6-element arrays such as CHARA or NPOI can measure only 15 baselines per spectral channel. Furthermore, for geo-synchronous targets one cannot use "Earth rotation synthesis", the strategy used with astronomical targets to exploit the rotation of the Earth to increase the instantaneous $u v$ plane coverage. The working model for interferometric GEO imaging has thus been (see, e.g. [4]) to assume spectral multiplexing, so that a single array configuration can be used at multiple wavelengths simultaneously so as to "fill in" the $u v$ plane.

A quite different problem, which is the focus of this paper, is the fact that the short baseline lengths required for imaging the brighter, and hence largest, GEO targets are not available at existing long-baseline interferometers. The one-metreclass unit telescopes required for high sensitivity are not compatible with close-packed interferometer configurations, and so the shortest baselines that have been utilised to date have been $\sim 10 \mathrm{~m}$ in length. In the absence of such short, 1-10 m, baseline data, other techniques, such as application of positivity and support (i.e. field-of-view) constraints, can be used to assist in reliable image recovery. A more advantageous method may be to secure the necessary low spatial frequency samples of the Fourier plane with a more compact, but still sensitive, short-baseline interferometer.

In this paper we propose the use of a separate large diameter $\mathrm{AO}$-corrected telescope operating in an interferometric mode to secure these data. More specifically, we are proposing the use of a non-redundant aperture mask (NRM) in concert with a conventional $\mathrm{AO}$ system on a large telescope to secure the relevant short baseline interferometric measurements. The use of NRM methods for high-angular resolution interferometric imaging was first implemented over twenty years ago $[5,6]$ and has since become a relatively routine procedure. In this approach a pupil plane mask with a non-redundant configuration of sub-apertures is used to convert a single telescope into a multi-element interferometer (see, e.g. [7]).

Short-exposure images taken with such a set-up show the image of the target crossed by multiple sets of interference fringes, and a Fourier analysis of sets of such interferograms gives the identical $u v$ plane data that would have been secured with a separated-element interferometer with the configuration of telescopes defined by the aperture mask. Astronomical images derived from such experiments are diffraction-limited and frequently surpass the fidelity realised by AO-corrected filled-aperture telescopes (see, e.g. [8]) due to the additional control of the imaging process that the use of non-redundant pupil affords.

In order to reach an appropriate sensitivity level, we propose here the use of a non-redundant mask coupled with a conventional AO system. Such a hybrid approach was first investigated computationally in the 1990s $[9,10]$ but it was only much later that these ideas were validated onsky [11]. The benefits of the combination of NRM and AO are manifold and include the following:

- An aperture mask samples the low spatial frequencies of the Fourier plane in a very similar way to that of a long baseline interferometer. This facilitates the merging of the two interferometric datasets, allowing existing long baseline image recovery codes to be used straightforwardly;

- The use of a mask removes the redundancy of the pupil, limiting the so-called "atmospheric noise" arising from multiple physical baselines in the pupil contributing to any given spatial frequency. This can have a beneficial impact in reducing the variability of the AO-related transfer function;

- By removing spatial fluctuations in the incoming wavefronts, the AO system usefully increases the diameters of the sub-apertures that can be utilised in the non-redundant mask. This provides enhanced sensitivity;

- By freezing the atmospheric perturbations, the AO system delivers a further level of sensitivity by allowing longer exposure times to be used. Aperture masking experiments are usually undertaken with exposure times comparable to the atmospheric coherence time, $t_{0}$. A good AO system will allow an order of magnitude increase in this before any residual temporal fluctuations in the AO-corrected wavefronts 
render the high angular resolution information compromised.

These expectations have been realised in recent experiments at the Palomar 200 inch telescope where an aperture mask was used with the Palomar laser guide star (LGS) AO system [12] to study targets as faint as magnitude 12 in the near-IR $K$ band. This is at least a factor of 10 times fainter than the brightest GEO targets, and within a factor of two of the median brightness of the current population of geosynchronous satellites. As LGS AO systems become more effective, aperture masked observations of even fainter GEO targets will likely become possible, although in this regime, the sensitivity of the separated element array providing the long baseline data may become the limiting factor.

\section{IMAGING MODEL}

For the imaging simulations presented here we have followed similar procedures to our most recent study of the feasibility of synthesis imaging of geo-synschronous targets [2] in assuming the availability of a second-generation long baseline array with ten unit telescopes, and separate fringe-tracking and science beam combiners. The fringe tracker is used to monitor and control the atmospheric perturbations between nearest-neighbour pairs of telescopes, while the science beam combiner mixes the signals from all pairs of telescopes together so as to record interference fringes from all possible baselines in the array. The individual telescopes are assumed to be $1.4 \mathrm{~m}$ in diameter, i.e. just over a few times $r_{0}$ in size at $1.65 \mu \mathrm{m}$. This represents a useful compromise between maximising sensitivity and minimising the impact of spatial wavefront perturbations introduced by the atmosphere. The use of moderate sized telescopes with local fast tip-tilt correction can frequently reach sensitivity levels that are very close to those realised with arrays using much larger telescopes with sophisticated AO systems [13].

These interferometric system parameters closely match those planned for the MROI, currently under construction in southern New Mexico. This second generation facility array has been planned to deliver model-independent images of faint and complex astronomical sources and its design and planned implementation differs from other existing arrays in three key ways:

- It has been specified to have a sensitivity some 50 to 100 times better than current arrays, primarily through very challenging requirements on throughput and delivered wavefront quality. This has a direct bearing on its utility for geosynchronous target imaging;

- It aims to utilise a much larger number of telescopes (10) than is currently the case - typically only 4-6 are used routinely at most astronomical facility arrays - so as to sample the Fourier plane more rapidly and uniformly than is usual. This again is expected to have a major bearing on its potential role for imaging GEO targets where Earth-rotation synthesis is not possible;

- It had been designed with a large amount of automation, e.g. for internal alignment and pre-observation set-up, so as to make data collection as efficient in time as possible. The ability to secure data in a time interval that is short compared to the time for the target to change its orientation will be key for GEO observations.

We have assumed that all 10 array elements are available and that they are located in the same Y-shaped layout and with identical inter-telescope separations as for the most compact MROI configuration. This is depicted in figure 1
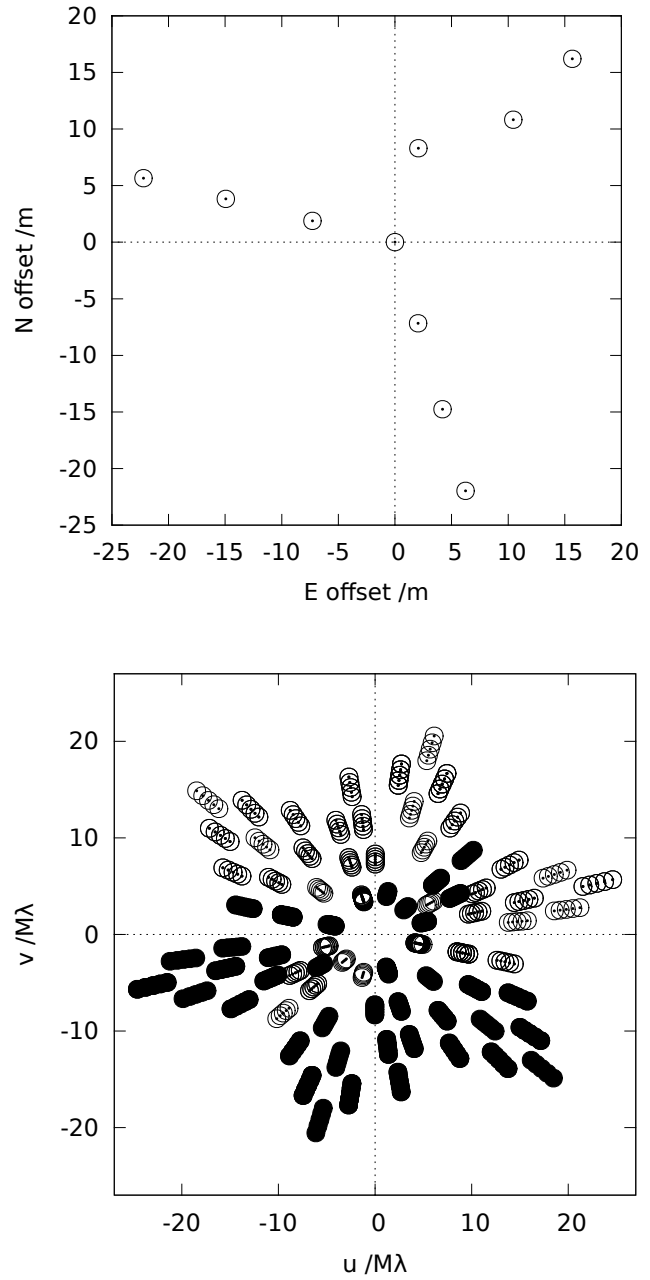

Figure 1. Array layout (top) and Fourier plane coverage (bottom) of the MROI in its most compact configuration. In the lower panel the radial "zoom" in the sampling is associated with the five spectral channels that have been assumed across the near-IR $H$ band at $1.65 \mu \mathrm{m}$. The empty region at the very center of the lower Fourier plane coverage plot is associated with the lack of baselines shorter than $8 \mathrm{~m}$ in the array layout. It is this region that the single dish telescope data populate.

which also shows the instantaneous $u v$ plane coverage for a target at the zenith. In this configuration, the shortest interferometer baseline is $7.8 \mathrm{~m}$, while the maximum baseline is approximately $42 \mathrm{~m}$. With a total of 10 telescopes, there will be 45 instantaneous baselines, and with an assumed 5 spectral channels across the near-IR $H$ band, this implies a sampling of the Fourier plane at a total of 225 independent $u v$ co-ordinates. This is perhaps not as many as one might favour, but is representative of what can probably be realised in the near term. Unlike our earlier preliminary study [2] where observations at $2.2 \mu \mathrm{m}$ were presented, we have chosen here to model a situation where the imaging data are secured at $1.65 \mu \mathrm{m}$ while the atmosphere above the array is monitored at $2.2 \mu \mathrm{m}$. This is attractive for two reasons: first, the science data will have $30 \%$ better angular resolution than at $2.2 \mu \mathrm{m}$, and second, the atmospheric monitoring will be improved because the object will be less resolved at longer wavelengths and hence the fringe contrasts in the fringe-tracking channels will in general be greater. 

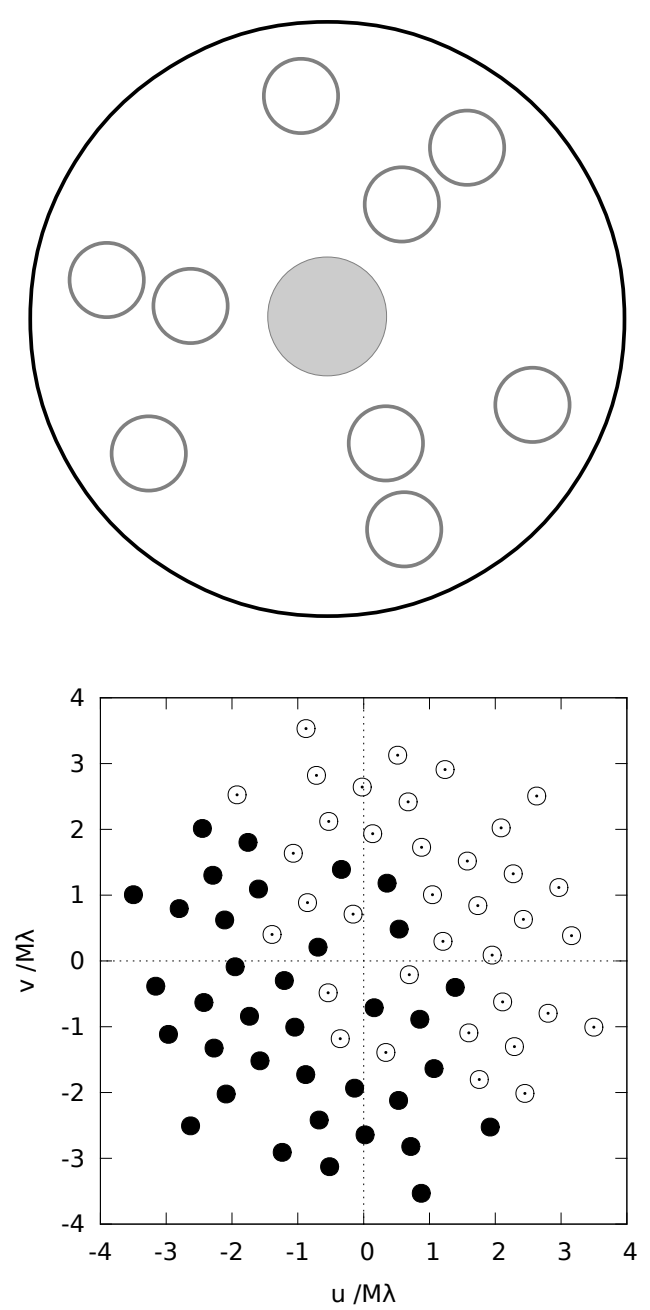

Figure 2. Mask layout (top) and associated Fourier plane coverage at $1.65 \mu \mathrm{m}$ (bottom) for a 9-hole non-redundant mask. The filled region in the center of the upper panel represents the typical secondary obscuration of a typical $8 \mathrm{~m}$ diameter telescope. The mask has sub-apertures of $1 \mathrm{~m}$ diameter and shortest and longest inter-sub-pupil distances of $1 \mathrm{~m}$ and $6 \mathrm{~m}$ respectively. The Hermitian-symmetric copies of a given Fourier data point are shown in the lower panel as filled circles and circles with dots in the centre respectively. Note that all the spatial frequencies fit within the central "empty" core of the Fourier plane coverage shown in figure 1.

As far as the short baseline data are concerned, we have assumed the use of an $8 \mathrm{~m}$ single dish telescope with a moderate order AO system feeding corrected wavefronts through a 9hole non-redundant mask to a fast-readout near-IR camera. The mask provides good 2-dimensional $u v$ plane coverage with baseline lengths ranging from just over 1 metre to around 6 metres. In good seeing conditions (i.e. 0.7 seconds of arc), $r_{0}$ will be approximately $60 \mathrm{~cm}$ in the $H$ band, and so we have assumed a conservative sub-aperture diameter of $1 \mathrm{~m}$. A schematic illustration of the non-redundant mask is shown in figure 2 which also shows its corresponding $u v$ plane coverage.

While the short and long baseline interferometric data need not be collected simultaneously, it will be important that any time interval between them being secured is small compared to the time with which a GEO target may change its ori- entation significantly with respect to the observers and its illumination. The MROI design goal is to allow capture of target data on its 45 instantaneous baselines within 10 minutes, after which a similar period of time will be needed for calibration observations on unresolved targets. We have assumed that this is fast enough that changes in the target orientation will not be an issue. This timescale is consistent with the time needed to secure the aperture-masked data for which perhaps several hundred $\sim 1$ second interferograms will be required. In a similar vein, it is not required that the single telescope and the long baseline interferometer be co-located, although the illuminations and orientations of the target must be similar enough that the data can be fused on the assumption of an identical brightness distribution at the angular resolution of the long baseline array.

For both the long and short baseline data we assume that the basic observables are the power spectrum and bispectrum of the target brightness distribution. While the use of a perfect AO system on a single telescope might allow true Fourier phases to be recovered from individual interferograms, in practice this is likely to be corrupted by residual sub-aperture "piston" phase errors. Closure phases are used here because they are much more robust against these types of residual wavefront perturbations.

Where the long and short baseline data will differ most will be in the assumed detector integration time. For the long baseline measurements, we have assumed an observing scenario where slow, and hence sensitive, group delay fringe tracking is performed in the $2.2 \mu \mathrm{m} K$ band, and where the high resolution science data are secured using fast $2 t_{0}$ integrations so as to freeze the seeing at $1.65 \mu \mathrm{m}$. For the AO-corrected NRM observations we have assumed a much longer exposure time of one second, i.e. we have taken as a given that the LGS AO system can maintain lock for at least this amount of time. Under these circumstances, and with nine $1 \mathrm{~m}$ diameter sub-apertures feeding light to the telescope focal plane, we anticipate that readout noise on the camera will not be a limiting factor in compromising the interferometric data.

\section{Simulations}

Simulations were performed for two representative GEO target morphologies to assess the impact of low spatialfrequency sampling of the Fourier plane for sources with different overall shapes and aspect ratios. Truth images were prepared using the TASAT software [14] and scaled so as to mimic one target with a moderate sized (i.e. few metre long) bus but with a pair of long and thin solar panels spanning $27 \mathrm{~m}$, and another with a larger bus and a single solar panel at one of its ends, with the panel having a largest dimension of $15 \mathrm{~m}$. The large sizes of these targets suggest that they would be relatively bright objects, and so we have prepared simulated datasets for two different brightness levels, one corresponding to a $1.65 \mu \mathrm{m}$ magnitude of 8 , and the other for a target six times fainter $\left(m_{H}=10\right)$ which is marginally brighter than the median GEO target in this waveband.

The interferometric datasets were generated by computing the Fourier transforms of the truth images at the spatial frequencies corresponding to the assumed MROI interferometric projected baselines and those sampled by the non-redundantly masked single telescope. The targets were assumed to have a constant structure across the near-IR $H$-band window: this is likely to be a valid assumption since this window only 
extends from $1.5 \mu \mathrm{m}$ to $1.8 \mu \mathrm{m}$. In order to best mimic existing interferometric experiments, the long-baseline data were computed for five separate narrow-band channels across the $H$ band, while the shorter baseline single telescope measurements were assumed to be broad-band single channel measurements.

These Fourier data were then used to calculate noise-free values for the relevant interferometric observables, i.e. squared visibilities and bispectra, and these were then corrupted so as to mimic realistic observations for the two target brightnesses under study. Experience has shown that most aperture masking experiments are usually limited by calibration errors, and so for the results presented here we have included significant calibration errors on the short baseline power spectra and bispectra. Fractional errors of $10 \%$ were assumed for $\Delta V^{2} / V^{2}$ while $5^{\circ}$ errors were added to the closure phases. These are much larger than is typical for bright-source NRM observations, but since no reports of AO-assisted NRM imaging of GEO targets exist in the open literature, we felt it would be prudent to use very conservative numbers here. For the long baseline array data more detailed modelling of system performance is available and so we used the MROI error budget to compute the signal-to-noise expected for a switchable 6-beam image plane $H$-band beam combiner using a detector with $4 \mathrm{e}^{-}$readout noise and subject to the residual atmospheric and instrumental coherence losses expected for the assumed target brightnesses and good seeing of 0.7 second of arc. Further errors were added to account for imperfect calibration of the interferometric transfer function, these being at the level of $\Delta V^{2} / V^{2}=0.02$ for the Fourier amplitudes and $0.8^{\circ}$ for the closure phases. Due to the switchable beam combiner architecture, five successive configurations of the fast optical switchyard feeding the combiner were assumed, yielding measurements on $5 \times 15=75$ baselines, each at five spectral wavebands simultaneously. Hence the simulated MROI datasets comprised a total 375 Fourier data at 225 unique coordinates in the $u v$ plane.

The simulated squared visibilities and bispectra were saved in the OIFITS format [15] and then used as input to the BSMEM image reconstruction code [16]. The OIFITS standard allows for the inclusion of multiple sets of Fourier data, with associated errors, all of which can correspond to different samplings of the Fourier plane and different types of observables. Here, our OIFITS files either contained the long baseline array power spectra and bispectra alone, or also included the short baseline power spectrum and bispectrum measurements sampled by the non-redundant mask. All the data are treated equivalently by the BSMEM code in the sense that it simply aims to determine the sky brightness distribution most consistent with the superset of the data and their errors.

We followed a very similar strategy for the reconstructions as before, employing a two-step scheme that we have used in the past for our entries to the IAU-sponsored beauty contests [17]. First, the highest spatial frequencies - corresponding to the noisiest data - were removed and BSMEM was run with a circular Gaussian prior image. The resulting preliminary image was then low-pass filtered by convolving it with a circular Gaussian and any pixel values below a userselected threshold were set to zero. The resulting image was then used as the prior for a second run of BSMEM on the full dataset, with no additional user intervention so as to mimic as unbiased an observation and reconstruction as possible.

\section{RESULTS}

Figure 3 shows the results of our image reconstructions for the target with a pair of $11 \mathrm{~m}$ long solar panels in the "bright source" regime $\left(m_{H}=8\right)$ and whose longest dimension is $27 \mathrm{~m}$. In this and all the subsequent reconstructions presented here, the leftmost image shows the truth data smoothed to the diffraction limited resolution of the combined dataset, the central panel shows the reconstruction from the long baseline array data alone, and the right hand panel is the image recovered where the additional short spacing data from the nonredundant aperture masked telescope have been included. The value of the short baseline measurements is immediately apparent from figure 3. While the image recovered from the long baseline data shows the correct orientation of the solar panels and the presence of a central bus, much of the finer detail in the reconstruction has appeared in the wrong place. Despite the high signal-to-noise ratio of the data, the solar panels appear very different in brightness and shape to each other and the bus appears to be split into two parts. Furthermore, there is noticeable background noise across the whole field of view defined by the prior image used for the second stage of the reconstruction procedure.

The image recovered from the combined short and long baseline data (right hand panel of figure 3) is considerably more faithful to the actual image. Not only are the solar panels reproduced faithfully, but many of the finer details of the satellite bus are clear, and there is very little spurious off-target noise. This type of behaviour is evident at fainter target brightnesses too (see figure 4). As before, the image recovered from the long baseline data alone is barely useful, while the use of the shortest interferometric spacings allows for the correct orientation of the target's solar panels to be visualised as well as the basic structure of satellite bus to be assessed. The much lower signal-to-noise of the data undoubtedly has an impact on the imaging fidelity, but the low level of spurious noise in the field of view is very evident.

The extent to which these initial results are a function of the target morphology can be assessed by examining the results shown in figures 5 and 6 . Here, the target does not have a single predominant axis in its brightness distribution and its flux is distributed over multiple large two-dimensional structures. This presents a much more challenging problem for any image reconstruction procedure that has access to only limited regions of the Fourier plane, and this was confirmed by our simulations. Even in the high light, and hence high signal-to-noise, regime it was impossible to recover a reliable image from the long baseline interferometer data alone (central panel of figure 5). The resulting images depended sensitively on the choice of parameters for the blur and threshold used in the initial reconstruction step, and in no case were we able to recover an image that correctly captured even the basic structure, i.e. an offset bus with a single large rectangular solar panel, of the target.

As soon as the short baseline NRM data were included, immediate benefits in performance were realised. The overall satellite morphology was faithfully reconstructed, and much of the finer detail seen in the truth image was discernible, including the circular antennae, the diagonal shadow on the bus and the grid pattern on the solar panel. Furthermore, the reconstructions were robust to changes in the starting point and parameters of the imaging procedure and so routinely converged to the same final image. Overall, the imaging fidelity was comparable to that achieved with the first target. At lower signal levels - once again, we repeated the simulations for a target six times fainter (see figure 6) - the 

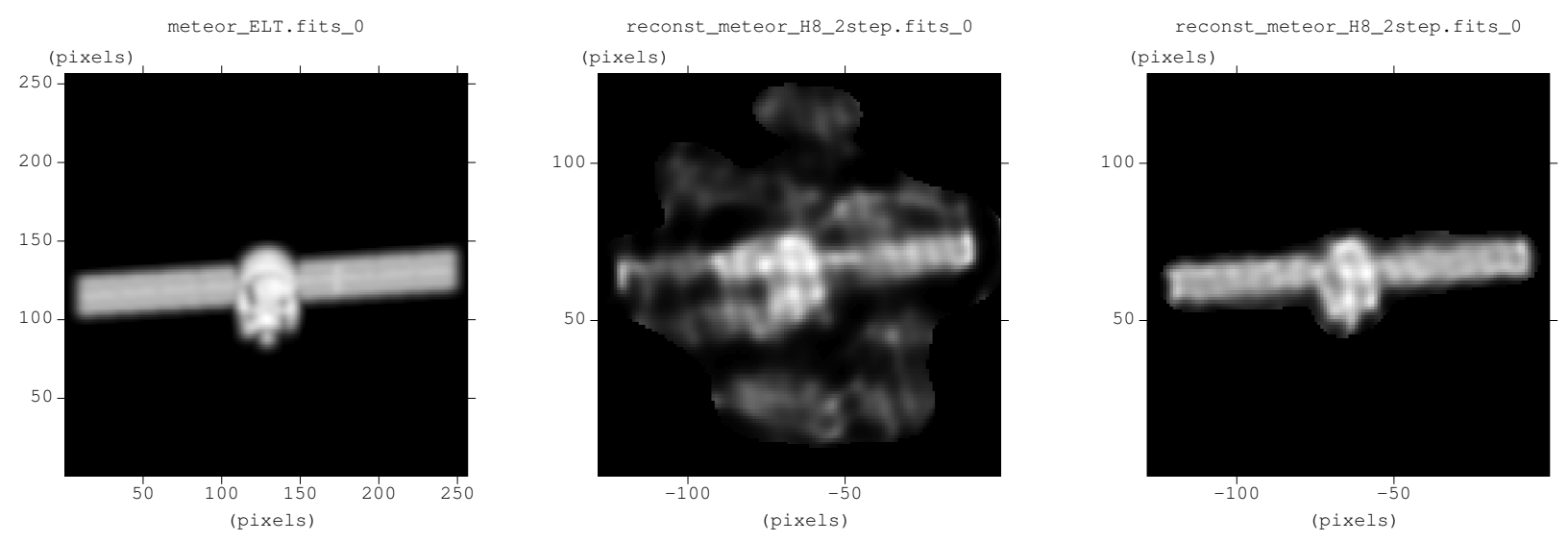

Figure 3. Truth image (left) and interferometric image reconstructions for a bright, $m_{H}=8$, GEO target with an extent of $27 \mathrm{~m}$ in its longest dimension. In the middle panel, only the long baseline data obtained with a 10-element MROI-type have been assumed, whereas in the right-hand panel additional data secured with a non-redundantly masked $8 \mathrm{~m}$-class telescope have been included. The increase in imaging fidelity when short baseline measurements are available is very obvious. The angular resolution of the truth image is 8 milliarcseconds, which corresponds to roughly $1.4 \mathrm{~m}$ at geo-synchronous altitude.
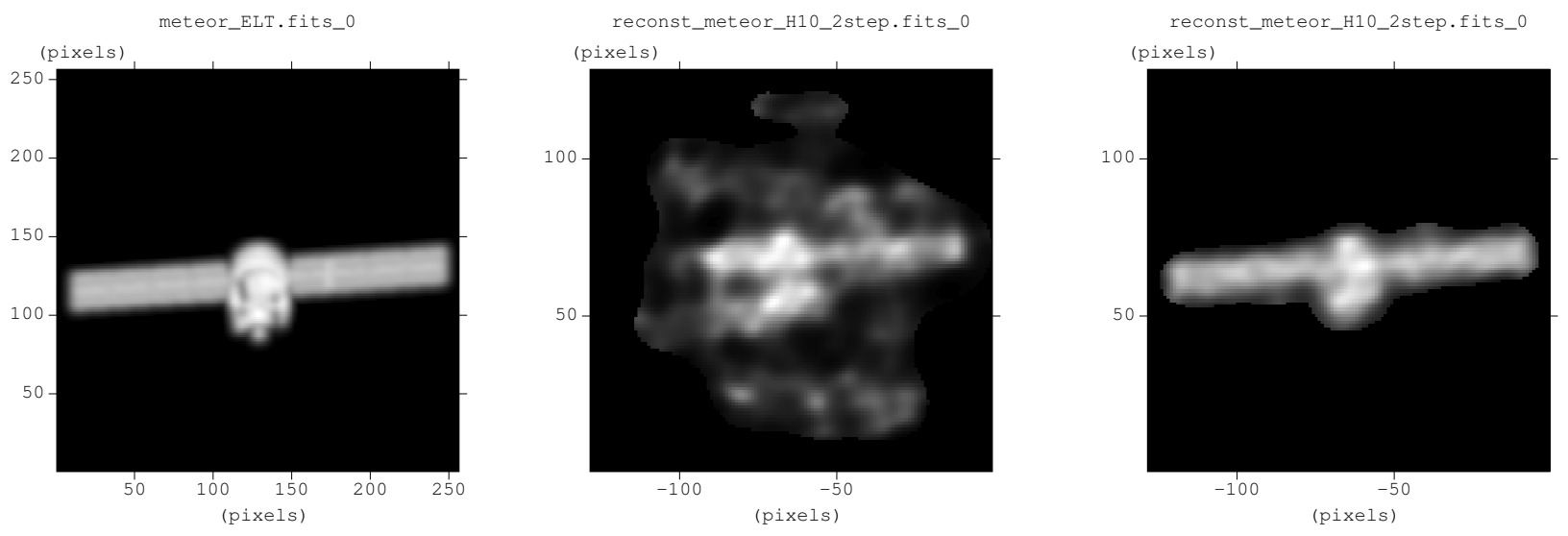

Figure 4. Truth image (left) and interferometric image reconstructions for the same target as for figure 3 but for a source brightness roughly six times fainter $\left(m_{H}=10\right)$. As before, the middle panel shows the image recovered from data secured on interferometric baselines from $8 \mathrm{~m}$ to $42 \mathrm{~m}$, while the rightmost panel shows the type of improvement realised if additional interferometric measurements secured on 1-6 $\mathrm{m}$ baselines are provided to the image reconstruction code.

main impact was to lower the signal-to-noise ratio on the long baseline data, and hence reduce the level and fidelity of the fine detail in the reconstructions. In the absence of the short baseline data, these fine details frequently appeared at quasirandom locations over the field of view, and as before it was impossible to identify even the most rudimentary large scale features of the target. The combined dataset was much more useful, showing both the large and smaller structures in the source, albeit with poorer angular resolution than at higher light levels.

\section{Discussion}

The results of our simulations reveal quite clearly the importance of short baseline measurements, that is, low angular resolution data, for high fidelity imaging of large geosynchronous satellites. The results presented here should be contrasted with those in our previous paper [2] which demonstrated that a separated-element interferometer alone can image slightly smaller satellites $(\lesssim 24 \mathrm{~m})$, if the observations are performed at a longer wavelength $(2.2 \mu \mathrm{m}$ versus $1.65 \mu \mathrm{m})$.

We note that these simulations (as well as our earlier paper) support the idea that particularly dense or high-signalto-noise coverage of the low spatial frequencies is not required for successful imaging of satellites, which have relatively simple large-scale structure. Additional simulations (not shown) with smaller calibration errors on the aperturemasking data did not exhibit any appreciable improvement in the quality of the reconstructed images. Although the assumptions made here about the accuracy of the NRM calibration are probably very conservative, our experience with these additional simulations suggests that had we assumed the use of better calibrated data, this would likely not have altered our assessment of the viability of interferometric imaging of GEO targets using our proposed scheme. We do not expect 

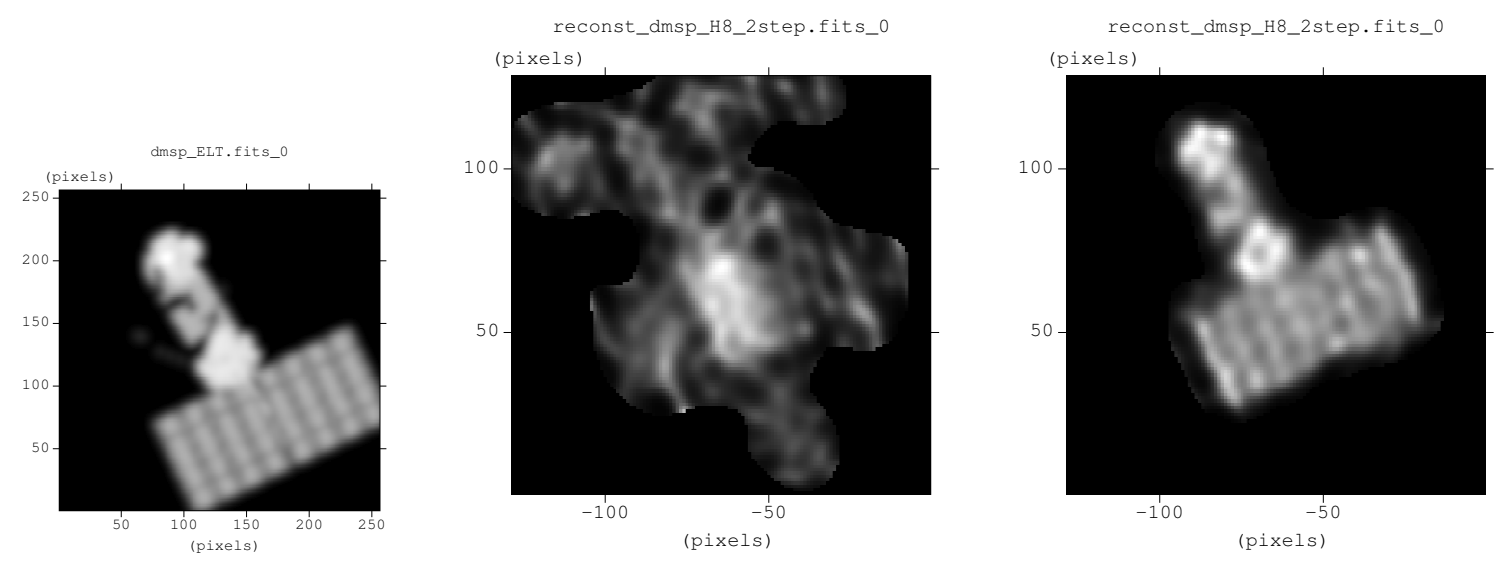

Figure 5. Truth image (left) and interferometric image reconstructions for a bright $\left(m_{H}=8\right)$ assymetric GEO target. The bus + payload on this target is of comparable size to the single solar panel, for which the longest dimension is $15 \mathrm{~m}$. The middle panel shows the image recovered from data on interferometric baselines from $8 \mathrm{~m}$ to $42 \mathrm{~m}$, while the rightmost panel shows the significant improvement realised if additional interferometric measurements on $1-6 \mathrm{~m}$ baselines are included.
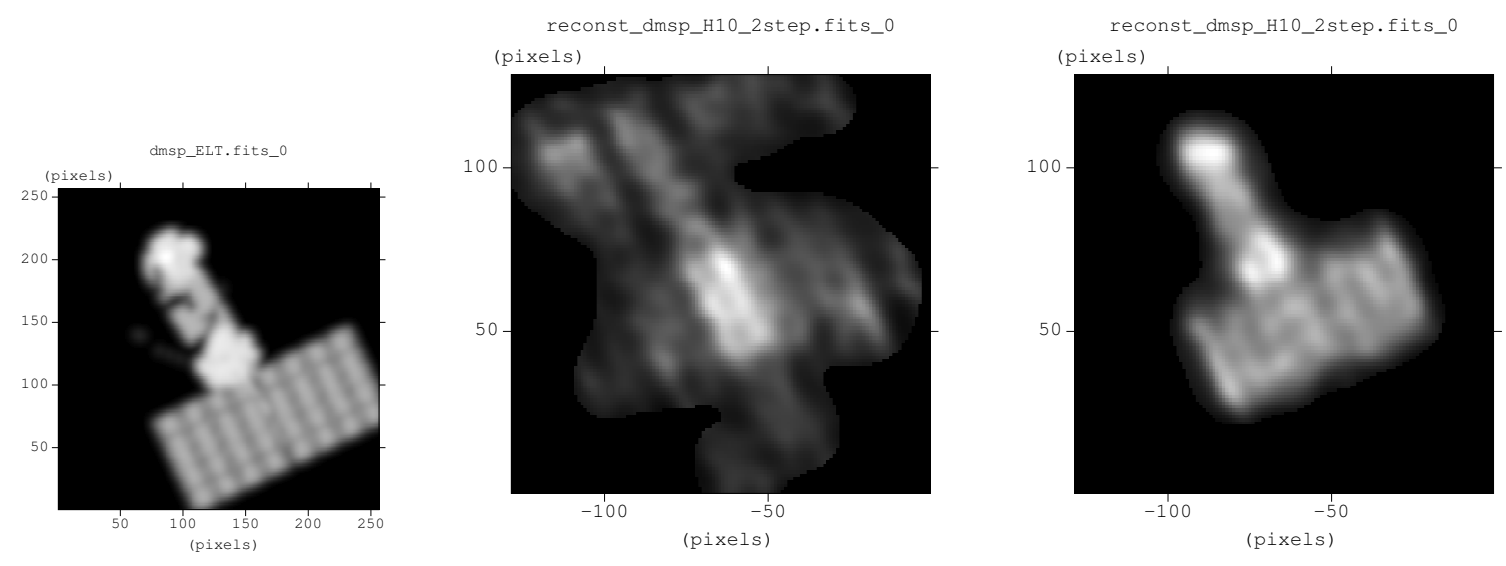

Figure 6. Truth image (left) and interferometric image reconstructions for the same target as for figure 5 but for a target brightness roughly six times fainter $\left(m_{H}=10\right)$. As before, the middle panel shows the image recovered from data secured on interferometric baselines from $8 \mathrm{~m}$ to $42 \mathrm{~m}$, while the rightmost panel shows the result obtained when additional measurements on $1-6 \mathrm{~m}$ baselines are included.

that using larger numbers of sub-pupils would have given a significantly enhanced capability either for the sizes of satellites considered here.

The factors that limit the quality of the images obtained from the combination of NRM and interferometer array data are the signal-to-noise ratio of the long-baseline data (where the target is most resolved), the remaining small gaps in the Fourier plane coverage (see figure 1), and the lack of stilllonger baselines. All of these combine to limit the amount of fine detail that can be recovered.

The signal-to-noise ratio issue probably has the greatest impact, as illustrated by the difference between the results for $m_{H}=8$ and $m_{H}=10$. A new generation of near-infrared APD array detectors from companies such as SELEX offers the prospect of sub-electron readout noise, which would give a significant improvement in the signal-to-noise ratio on long baselines compared with the $4 \mathrm{e}^{-}$noise assumed for these simulations. Adoption of these detectors would also enable useful observations of faint targets in the $J$ band $(1.25 \mu \mathrm{m})$, which could be combined with $H$ band data to extend the Fourier coverage to deliver $30 \%$ higher angular resolution.

We have shown that aperture-masked observations at a large AO-equipped conventional telescope can in principle be combined with measurements from a separated-element interferometer to realise large improvements in imaging quality. However, there are significant practical obstacles to securing such data, which we do not wish to understate. The observations must be secured at locations and times such that both the illumination of the satellite by the sun and the apparent orientation of the solar panels (which rotate to track the Sun) are comparable, and signal-to-noise considerations demand above-average seeing at both telescope sites. The observations could be obtained on different nights (on the assumption that no material change to the satellite had occurred), which ameliorates the scheduling issues somewhat. On the other hand, our proposed approach makes use of general purpose astronomical facilities and hence is likely to be far more cost- 
effective than building a dedicated telescope or interferometer for observations of GEO targets alone.

\section{SUMMARY}

With the emergence of sensitive optical/IR synthesis arrays such as the MROI, optical interferometric imaging will potentially offer an important capability for space situational awareness, allowing satellites in geo-synchronous orbit to be identified and apparent damage to be assessed. We have presented results which illustrate the impact of a lack of short baselines on the quality of the images reconstructed from interferometer data, as well as the viability of a solution to this problem for large ( $\gtrsim 20 \mathrm{~m}$ ) satellites, namely the incorporation of interferometric data obtained by non-redundant masking of an AO-equipped 8-10 $\mathrm{m}$ telescope.

The application of the proven NRM technique to GEO imaging, in combination with separated-element interferometry, offers a number of advantages over the use of an interferometer array alone:

- Imaging of the larger half of the GEO size distribution is enabled;

- Since larger satellites are brighter, the chance of developing a successful capability is greatly improved;

- The need for using strong prior information (which would otherwise be required in difficult cases) in the reconstruction process is eliminated.

Most importantly, the results presented here conclusively demonstrate that a useful quality and fidelity of imaging can be obtained for GEO targets of a realistic size and brightness, paving the way for further investigation of the technical challenges associated with these types of observations.

\section{ACKNOWLEDGMENTS}

The authors thank the UK's STFC for continued support for work on high angular resolution imaging methods at the University of Cambridge, and their colleagues at New Mexico Tech for encouraging this study.

\section{REFERENCES}

[1] Creech-Eakman, M.C., Romero, V., Payne, I., et al., "The Magdalena Ridge Observatory Interferometer: a status update", 2012, Proc. SPIE 8445, 84450P84450P-10

[2] Young, J.S., Haniff, C.A., Buscher, D.F., CreechEakman, M., Payne, I., Jurgenson, C.A., Romero, V., "The MROI's capabilities for imaging geosynchronous satellites", 2012, Proc. SPIE 8445, 84452N-84452N-11

[3] Mozurkewich, D., Armstrong, J.T., Hindsley, R.B., Jorgensen, A.M., Restaino, S.R., and Schmitt, H.R., "An imaging interferometer for compact sources", 2012, Proc. SPIE 8165, 81650X-81650X-10

[4] Schmitt, H.R., Mozurkewich, D., Restaino, S.R., Armstrong, J.T., Hindsley, R.B., and Jorgensen, A.M., "Simulated optical interferometric observations of geostationary satellites", 2011, Proc. SPIE 8165, 81650T81650T-14

[5] Haniff, C.A., Mackay, C.D., Titterington, D.J., Sivia,
D. and Baldwin, J.E., "The first images from optical aperture synthesis", 1987, Nature 328, 694-696

[6] Buscher, D.F., Baldwin, J.E., Warner, P.J. and Haniff, C.A., "Detection of a bright feature on the surface of Betelgeuse", 1990, Mon. Not. R. ast. Soc. 245, 7P-11P

[7] Tuthill, P.G, Monnier, J.D., Danchi, W.C., Wishnow, E.H. and Haniff, C.A., "Michelson Interferometry with the Keck I Telescope", 2000, P.A.S.P. 112, 555-565

[8] Monnier, J.D, Tuthill, P.G., Lopez, B., Cruzalebes, P., Danchi, W.C. and Haniff, C.A., "The Last Gasps of VY Canis Majoris: Aperture Synthesis and Adaptive Optics Imagery", 1999, Ap. J. Letters 512, 351-361

[9] Nakajima, T. and Haniff, C.A., "Partial adaptive compensation and passive interferometry with large groundbased telescopes", 1993, P.A.S.P. 105, 509-520

[10] Haniff, C.A. and Wilson, R.W., "Closure-phase imaging with partial adaptive correction", 1994, P.A.S.P. 106, 1003-1014

[11] Lloyd, J. P., Martinache, F., Ireland, M.J., Monnier, J.D., Pravdo, S.H., Shaklan, S.B. and Tuthill, P. G., Direct Detection of the Brown Dwarf GJ 802B with Adaptive Optics Masking Interferometry", 2006, Ap. J. Letters 650, L131-L134

[12] Bernat, D., Bouchez, A.H, Ireland, M. et al., "A Close Companion Search Around L Dwarfs Using Aperture Masking Interferometry and Palomar Laser Guide Star Adaptive Optics", 2010, Ap. J. 715, 724-735

[13] Rea, A.D. and Haniff, C.A., "Enhancing the limiting sensitivity of optical/infrared interferometry with natural guide star adaptive optics: happy couples or bad bed fellows?”, 2012, Proc. SPIE 8445, 84451Q-84451Q-14

[14] Riker, J.F. and Butts, R.R., "The time-domain analysis simulation for advanced tracking (TASAT) approaches to compensated imaging", 1992, Proc. SPIE 1688, 548560

[15] Pauls, T.A., Young, J.S., Cotton, W.D. and Monnier, J.D., "A data exchange standard for optical (visible/IR) interferometry", 2005, P.A.S.P. 117, 1255-1262

[16] Baron, F. and Young, J.S., "Image reconstruction at Cambridge University", 2008, Proc. SPIE 7013, 70133X-70133X-11

[17] Malbet, F., Cotton, W., Duvert, G., et al., "The 2010 interferometric imaging beauty contest", 2010, Proc. SPIE 7734, 77342N-7734N-12

\section{BIOGRAPHIES}

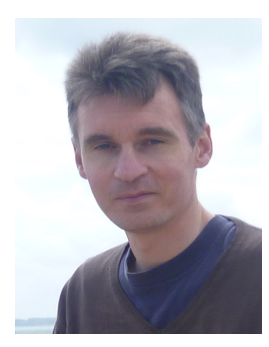

John Young is a Senior Research Associate at the University of Cambridge, $U K$. His research interests include the scientific exploitation of ground based interferometers, the design of interferometric instrumentation and the architecture and deployment of real-time and data analysis software for these instruments. Dr Young has particular expertise in image reconstruction from interferometric data, and has won the SPIE's biennial "Interferometric Imaging Beauty Contest" on three occasions in the past 10 years. 


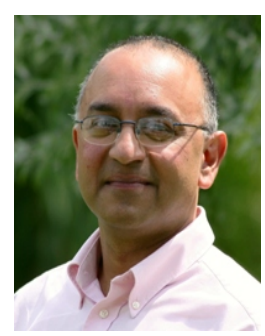

Chris Haniff is a Professor in Physics at the University of Cambridge, UK. He leads the Optical Interferometry group at the Cavendish Laboratory there, and is joint System Architect for New Mexico Tech's Magdalena Ridge Observatory Interferometer. His research has focused on the development of high angular resolution instrumentation and techniques for optical/IR astronomy. He has a bachelors degree in Physics and a PhD in Astronomy, both from the University of Cambridge.

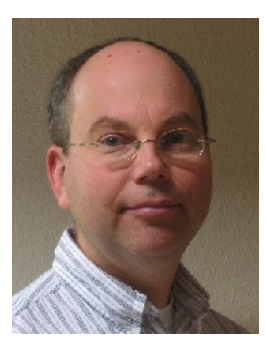

David Buscher is a Lecturer in Physics at the University of Cambridge, UK. He is joint System Architect for the Magdalena Ridge Observatory Interferometer in New Mexico. His work centers around designing, building, and using high-angular-resolution imaging instruments for optical and infrared astronomy. He has bachelors and PhD degrees bridge. in Physics from the University of Cam- 